\title{
A substorm in midnight auroral precipitation
}

\author{
V. G. Vorobjev ${ }^{1}$, O. I. Yagodkina ${ }^{1}$, G. V. Starkov ${ }^{1}$, and Ya. I. Feldstein ${ }^{2}$ \\ ${ }^{1}$ Polar Geophysical Institute, Russian Academy of Sciences, Apatity, Murmansk region, 184200, Russia \\ ${ }^{2}$ IZMIRAN, Russian Academy of Sciences, Troitsk, Moscow region, 142190, Russia
}

Received: 18 April 2002 - Revised: 17 March 2003 - Accepted: 13 May 2003

\begin{abstract}
DMSP F7 spacecraft observations for the whole of 1986 were used to construct the empirical model of the midnight auroral precipitation during a substorm. The model includes the dynamics of different auroral precipitation boundaries and simultaneous changes in average electron precipitation energy and energy flux in different precipitation regions during all substorm phases, as well as the IMF and solar wind plasma signatures during a substorm. The analysis of the model shows a few important features of precipitation. (1) During the magnetic quietness and just before the beginning of the substorm expansive phase the latitudinal width of the auroral precipitation in the nightside sector is about $5-6^{\circ} \mathrm{CGL}$, while that of the auroral oval is about $2-3^{\circ}$ CGL during such periods. (2) For about 5 min before the substorm onset a decrease in the average precipitating electron energy in the equatorward part of auroral zone was observed simultaneously, with an increase in both the average electron energy and energy flux of electron precipitation in the poleward part of the auroral zone. (3) The isotropy boundary position in the beginning of the substorm expansive phase coincides well with the inner edge of the central plasma sheet. The analysis of interplanetary medium parameters shows that, on average, during the substorm development, the solar wind dynamic pressure was about 1.5 times that of the magnetic quietness period. Substorms occurred predominantly during the southward IMF orientation, suggesting that substorm onset often was not associated with the northern turn or decrease in the southward interplanetary $B_{z}$. The Northern Hemisphere's substorms occurred generally during the positive interplanetary $B_{y}$ in winter, and they were observed when the interplanetary $B_{y}$ was negative in summer.
\end{abstract}

Key words. Ionosphere (auroral ionosphere; particle precipitation) - Magnetospheric physics (storm and substorm; magnetosphere-ionosphere interaction)

Correspondence to: V. G. Vorobjev

(vorobjev@pgi.kolasc.net.ru)

\section{Introduction}

DMSP satellites database for the whole of 1986 was created to investigate different types of auroral precipitation features. It contains more than 35000 satellite passes through the auroral zone, almost around the magnetic local time. In more detail, the description of the database will be presented below, and hereby we would only like to note that the level of geomagnetic activities and the phase of geomagnetic disturbances were determined for each satellite pass. Such detailed information allows one to examine the dynamics of different auroral precipitation boundaries and variations in the average energy and energy flux of precipitating particles in different parts of the auroral zone during a magnetospheric substorm. The model description of spatial distribution and basic characteristics of electrons precipitating into the polar ionosphere give an evident notion about the various population source behavior in the distant magnetosphere during substorms, and can be used to estimate the precipitation energy and its influence on ionospheric conductivity and the chemistry of the upper atmosphere.

Sotirelis and Newell (2000) published the boundaryoriented model of the global configuration of electrons precipitating into the polar ionosphere based on DMSP spacecraft observations. An essential advantage of this model in comparison with the earlier published models (Spiro et al., 1982; Hardy et al., 1985) is not only large statistics (12 years of continuous observations) and rather high spatial resolution of global distributions, but also regulation of precipitating electron features for each satellite pass over the polar ionosphere relative to various auroral precipitation boundaries. Our database is based on more modest statistics (1 year of observations), but it has an advantage in that it contains, in addition to different auroral precipitation boundary co-ordinates, additional information including interplanetary medium conditions, magnetic activity indices and magnetospheric substorm phases. This information enables us to carry out more fine investigations and to frequently represent the obtained dependencies in an analytical form handy for 
further applications. Thus, Vorobjev et al. (2000) and Starkov et al. (2002) published the quantitative correlations between the location of different auroral precipitation regions in the midnight and midday sectors and the magnetic activity level in the auroral zone. This was not possible in all previous models (Spiro et al., 1982; Hardy et al., 1985). Sotirelis and Newell (2000) used the $b 2 i$ boundary, the proxy for the ion isotropy boundary, to infer the magnetotail stretching. It is difficult to apply these models to more detailed research, particularly during the substorm development. In the present paper the empirical model of a substorm in the midnight auroral precipitation is presented. The model contains the dynamics of different auroral precipitation boundaries during all substorm phases, together with simultaneous changes in the average electron precipitation energy and energy flux in different precipitation regions, as well as the IMF and solar wind plasma features during the substorm.

\section{Data used and technique of processing}

DMSP F7 spacecraft observations in the magnetic local time interval from 21:00 MLT to 24:00 MLT were used to study auroral precipitation features during substorms. The objects of investigation are $b 1 e, b 2 e, b 2 i, b 4 s, b 5 e$ and $b 6$ boundaries, according to the notation suggested by Newell et al. (1996) and Feldstein and Galperin (1996) for nightside precipitations. In this notation the boundaries were numbered so that the number of the boundary grows with the increase in its latitudinal location. Indexes $e$ and $i$ refer to the electron and ion precipitation boundary, respectively; $b 1 e$ is the "zero-energy" electron boundary. According to the results by Vorobjev et al. (2000), this boundary coincides well with the equatorward boundary of diffuse auroral luminosity.

$b 2 e$ The boundary where the electron average energy is neither increased nor decreased with latitude. It is assumed that this boundary is a proxy for the inner edge of the central plasma sheet. According to a statistical study (Vorobjev et al., 2000), $b 2 e$ coincides with the equatorward boundary of the auroral oval.

$b 2 i$ The latitude where the energy flux of ions has the maximum. This boundary, a proxy for the ion isotropy boundary, corresponds to the earthward edge of the magnetospheric cross tail current sheet (Newell et al., 1996, 1998).

$b 4 s$ The equatorward boundary of spatial structured electron precipitations (low correlation coefficient between the neighboring spectra).

$b 5 e$ The poleward boundary where an abrupt drop by a factor of at least 4 in the electron energy flux is observed. According to Vorobjev et al. (2000), this boundary coincides well with the poleward boundary of the statistical auroral oval. $b 6$ The poleward boundary of subvisual drizzle. It is supposed that $b 6$ frequently corresponds to the polar cap boundary.

In addition to standard information about different auroral precipitation boundary coordinates and particle precipitation characteristics, interplanetary medium parameters and indexes of geomagnetic activity and substorm phases were determined for each satellite pass through the auroral zone. The substorm phase was determined from 1-minute averaged variations of the $A E$ and $A L$ indexes at the time when the satellite encountered the $b 2 e$ boundary. It takes only $2-3 \mathrm{~min}$ for the spacecraft to pass the precipitation zone, from the $b 1$ to $b 6$ boundaries. Magnetic activities were divided into four classes: 0 - the magnetic quiet period $(|A L| \leq 40 \mathrm{nT})$ which consists of all quiet times, not only just those preceding a substorm; 1 - the growth phase of substorm; 2 - the substorm expansive phase; 3 - the recovery phase of substorm; 4 - another type of magnetic activity $(|A L|>40 \mathrm{nT})$. Moreover, each of the 1,2 and 3 phases was additionally divided into three equal temporary intervals, corresponding to the initial, middle and final stage of each substorm phase (subphases). Such detailed separation allows one to obtain three experimental statistical points for each phase and to investigate the precipitation boundary dynamics and auroral electron characteristics during the magnetospheric substorm development, in detail.

Observations obtained by the DMSP F7 spacecraft with an orbiting period of approximately $100 \mathrm{~min}$ were used for the investigation. The duration of a typical isolated substorm from the beginning of the growth phase to the end of the recovery phase is about $2-3 \mathrm{~h}$. That is why in the course of any substorm there were only $2-3$ DMSP passes occurring in different substorm phases. For this reason different points were related to different substorms. Our results will describe an average or synthetic substorm, consisting of three phases. Data belonging to the same stage of each substorm phase were summarized to determine the average level of auroral boundary locations, electron precipitations and interplanetary medium parameters. The average value of all quantities are shown in figures as a point in the center of the corresponding substorm subphase. The numbers of events per statistical cell are changed from 200-300 for different subphases to about 700 for the 0 and 4 classes.

Substorm phases were defined in conformity with the classical reports by Akasofu et al. (1966), Kokubun (1970), McPherron (1970), Iijima and Nagata (1972) and others. The general feature of the growth phase was a slow growth of the magnetic activity prior to the rapid growth during the substorm expansive phase. The beginning of a sharp magnetic activity level increase was determined as a substorm onset time. If the average duration of growth, expansive and recovery phases is about $45-60 \mathrm{~min}$, then the duration of each subphase is about $15-20 \mathrm{~min}$. To illustrate this point, the average $A L$ index values for different subphase are shown in Fig. 1 by a dashed line. In order to clarify the substorm phase and subphase selection, the model of the corresponding magnetic 


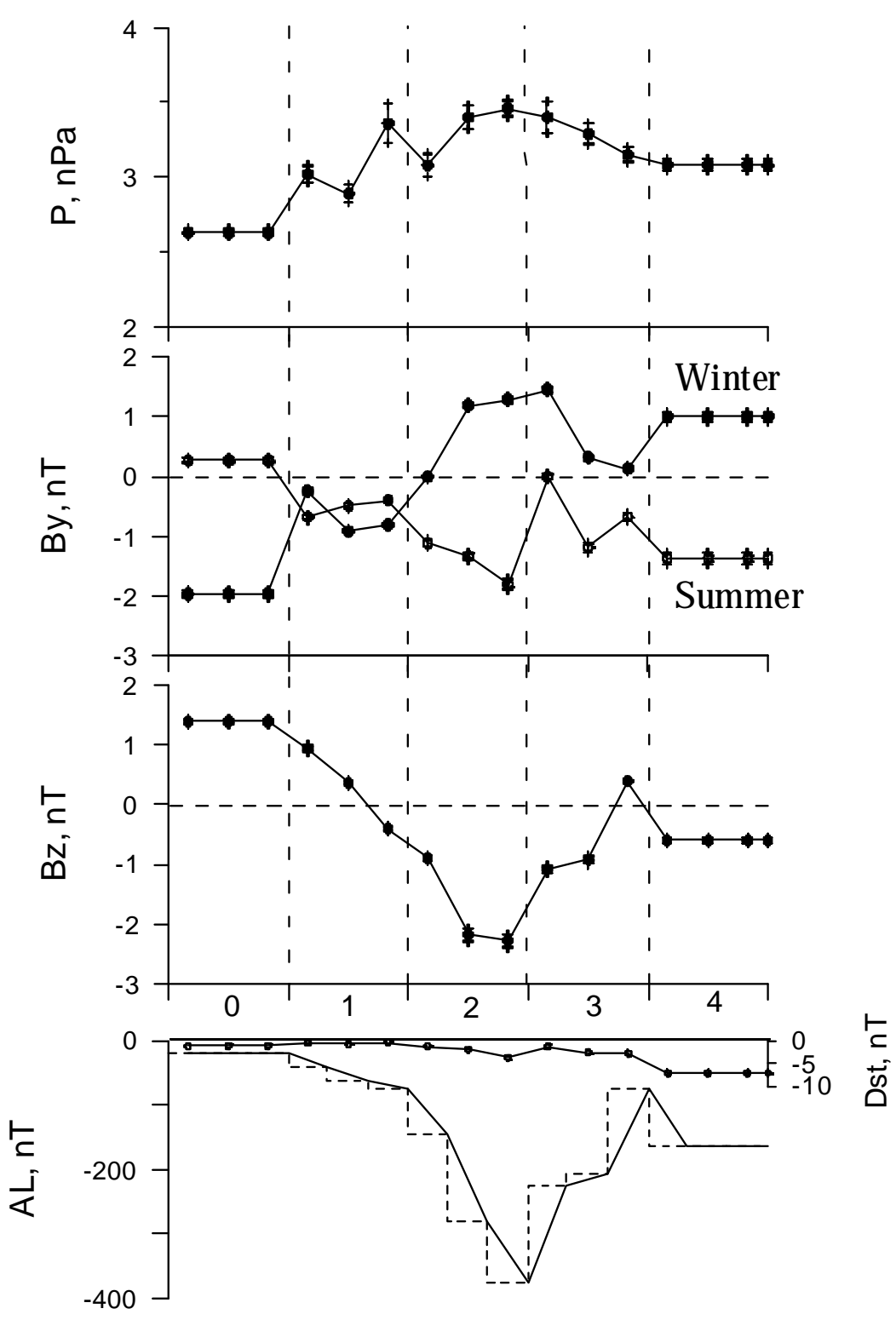

Fig. 1. Variations of interplanetary medium parameters during the magnetospheric substorm. From top to bottom are shown: the solar wind dynamic pressure $(P)$, variations of the IMF $B_{y}$ and $B_{z}$ components. Crosses show the standard deviation of measured quantities. The bottom panel illustrates the $A L$ and $D_{s t}$ variations. The phases of magnetic disturbance are marked along the horizontal axis.

disturbance shifted ahead in time by one-half of the substorm subphase, shown here by solid line.

\section{Observations and results}

The bottom part of Fig. 1 displays (indicated by the solid line) the variations of the $A L$ index during all magnetic activity intervals, which are marked along the horizontal axis. The curve is constructed by 5 -min averaged $A L$ indices, as an average $A L$ magnitude for satellite passes during the identical phase of magnetic disturbance. As it is seen from the figure that the shape of the $A L$ variation corresponds well to the typical magnetic substorm that testifies to rather a cor- rect determination of the magnetospheric substorm phases. The average level of magnetic activity during the period of the magnetic quietness was about $-20 \mathrm{nT}$. The magnetic activity gradually increased during the substorm growth phase, reaching about $-85 \mathrm{nT}$ just before the substorm onset. The average disturbance in the substorm maximum was $-410 \mathrm{nT}$, and during the disturbance period (4) without determined phase it was equal to about $-125 \mathrm{nT}$. In Fig. 1 the thin line with circles shows the changes in the $D_{s t}$ index during the same periods. The average variation of this index is very insignificant and even during the substorm maximum it does not exceed $-5 \mathrm{nT}$.

First, we examined the interplanetary medium parameters corresponding to each phase of magnetic disturbance. Solar 


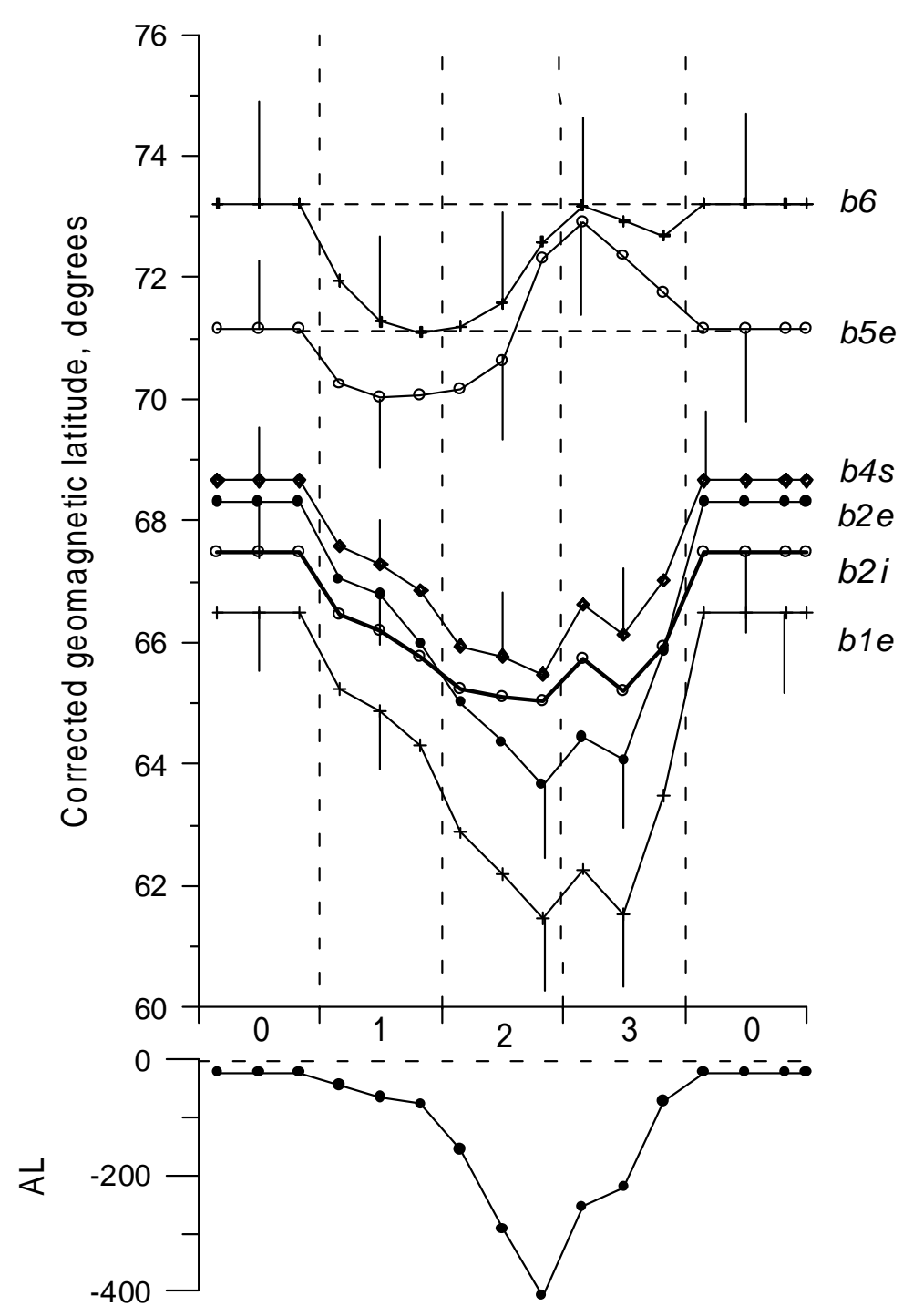

Fig. 2. The auroral precipitation boundaries during the substorm. The label of a boundary is marked in the right part of the figure. The root-mean-square errors are shown as half of a deviation to either side. The bottom panel shows the average $A L$ index variation.

wind plasma and IMF observations from the NSSDC OMNIWeb database were used. In three top panels of Fig. 1 the average value of the solar wind dynamic pressure $(P)$, and the IMF $B_{y}$ (separately for winter and summer seasons) and $B_{z}$ components are shown from top to bottom. Crosses in the figure show the standard deviation $(S)$ of the measured quantities: $S=\sigma N^{-1 / 2}$, where $\sigma$ is the rms deviation and $N$ is the number of events.

The analysis of interplanetary conditions demonstrates that, on average, the solar wind dynamic pressure was a little bit higher during the substorm than during the period of magnetic quietness. Substorms occurred predominantly during periods of southward IMF orientation, and substorm onsets were often not associated with the northern turn of the interplanetary $B_{z}$.

The winter of 1986 in this study is January, February and November, December, and the summer is May-August. Dis- tributions of the IMF $B_{y}$ occurrence frequency with a different sign and value during the summer and winter of 1986 are alike and approximately symmetrical, with a nearly equal occurrence of positive (46\% and $49 \%$ ) and negative (54\% and $56 \%$, respectively) IMF $B_{y}$. However, in winter, substorms were generally observed during the positive interplanetary $B_{y}$ and in summer they occurred during the negative $B_{y}$.

In order to take into account the time history of the solar wind, another method was also applied. We took the interplanetary parameters for the previous hour if the spacecraft pass occurred in the first half of any hour, and for the same hour if the pass took place during the second half of the hour. The results were qualitatively equal, and only a insignificant quantitative difference was observed.

The dynamics of different auroral precipitation boundaries during a substorm are illustrated in Fig. 2. On the horizontal 
scale in this figure the active period with an unidentified substorm phase (4) is replaced by the period of magnetic quietness (0). So, the disturbance under investigation corresponds to the averaged isolated substorm. The variation of the average $A L$ index value is shown in the bottom part of the figure. As the $D_{s t}$ variations in the examined interval were insignificant, it is possible to consider that all changes in the latitudinal location of the precipitation boundaries and auroral electron characteristics are caused generally by the substorm development.

Because of the relatively large statistical set, standard deviations $(S)$ are very small and actually slight for all quantities in our investigation. That is why in Fig. 2 the root-meansquare deviations were calculated for the boundary positions. In order not to overload the figure, these errors are shown as half of a deviation to either side.

Figure 2 shows that the equatorward precipitation boundaries $(b 1 e, b 2 e, b 2 i, b 4 s)$ move during both the growth and expansive substorm phases to lower latitudes and then return during the recovery phase to initial latitudes, correlating well with changes in the $A L$ index. The most equatorward boundary, $b 1 e$, shows the greatest equatorward displacement of about $5^{\circ}$ of corrected geomagnetic latitude (CGL), while the displacement of the $b 4 s$ boundary was about $2.5^{\circ}$ of the latitude. Higher latitude precipitation boundaries $(b 5 e, b 6)$ moved equatorward only during the substorm growth phase. During the expansive phase, the $b 6$ boundary returned to its undisturbed level, while the $b 5 e$ boundary moved further poleward by about $2^{\circ}$ of latitude higher than its location during the quietness period. Owing to such difference in the $b 5 e$ and $b 6 e$ boundaries dynamics, the region of soft precipitations between $b 5 e$ and $b 6$ shrank sharply during the expansive phase. At the maximum of the substorm the polar cap boundary (b6) approximately coincided with the poleward boundary of the statistical auroral oval $(b 5 e)$.

The $b 2 e$ boundary is more dynamic than the $b 2 i$. During the average substorm of about $400 \mathrm{nT}$ in intensity, the equatorward displacement of $b 2 e$ was about $5^{\circ}$ of the latitude, while it was only $\sim 2^{\circ}$ CGL for the $b 2 i$ boundary. During magnetic quietness, $b 2 e$ was placed poleward of $b 2 i$, i.e. the inner edge of the magnetospheric cross tail current sheet was closer to the Earth than the central plasma sheet (CPS). $B_{y}$ contrast, the isotropy boundary was observed inside the center plasma sheet during the expansive and recovery phases of the substorm. The position of the $b 2 e$ and $b 2 i$ boundaries approximately coincided around the substorm onset time. Such interdependence in the auroral boundaries position occurred not only statistically, but also for real individual disturbances. It is possible to suggest that the relative position of the $b 2 e$ and $b 2 i$ boundaries can testify to the quiet or disturbed condition of the magnetosphere.

In addition to the auroral precipitation boundary coordinates, the DMSP database contains the information about an average precipitating electron energy and energy flux in different precipitation regions. Taking into account that at midnight the position of the $b 1 e$ and $b 1 \mathrm{i}$ boundaries and the $b 5 e$ and $b 5 i$ boundaries, as a rule, coincide well (Newell et al., 1996; Vorobjev et al., 2000); such kind of data are available for four precipitation regions: $b 1 e-b 2 i-$ precipitations equatorward of the cross tail current sheet (diffuse auroral luminosity); $b 2 i-b 4 s$ - precipitations from the earthward edge of the current sheet (the equatorial part of an auroral oval); $b 4 s-b 5$ - precipitations in the poleward part of the statistical auroral oval, and $b 5-b 6$ - soft electron precipitation region poleward of the auroral oval. Precipitating electron characteristics in these regions are shown in Fig. 3. To compute these characteristics we have selected one more temporal interval in the magnetospheric substorm development. In the 1-3 phase, corresponding to the final stage of the substorm growth phase, we have chosen the satellite passes closest to the substorm onset, the time of which we have estimated as $\left(T_{0}-5\right) \pm 5 \mathrm{~min}$. Only events with the sharp pronounced magnetic bay onset were selected for this investigation.

In Fig. 3 the average electron precipitation energy and energy flux are standardized to those during magnetic quietness, whose levels in units $(\mathrm{keV})$ for the average energy $\left(E_{0}\right)$ and $\left(\mathrm{erg} / \mathrm{cm}^{2} \mathrm{~s}\right)$ for the energy flux $\left(F_{0}\right)$ are shown in the right part of Figs. 3a and b, respectively. Figure 3a illustrates changes in the average electron precipitation energy in different regions during the substorm. As seen from the figure during the substorm expansive phase, the average energy increased as large as $2-3$ times in all precipitation regions. The energy increase begins smoothly, yet during the substorm growth phase. The most interesting is the decrease in the average auroral electron energy in the two low-latitude regions ( $b 1-b 2 i$ and $b 2 i-b 4 s)$ and the simultaneous increase in the energy in the two higher latitude regions $b 4 s-b 5$ and especially in $b 5-b 6$, just before the substorm onset time. The energy decrease in the two low-latitude auroral regions can correspond to the fading of both discrete and diffuse aurorae prior to the substorm onset, as noted by Zaitseva et al. (1976), Pellinen and Heikkila (1978) and Kornilova et al. (1989).

Figure $3 \mathrm{~b}$ shows the behavior of the average energy flux in the same precipitation zones as the average energy in Fig. 3a. During the period of magnetic quietness, the greatest power emitting occurred in the $b 4 s-b 5$ region, corresponding to the poleward part of the statistical auroral oval. The precipitating electron energy flux in this region was about 3 times higher than in the equatorward part of the auroral oval ( $b 2 i-$ $b 4 s$ ) and more than ten times higher than in the $b 1 e-b 2 i$ and $b 5-b 6$ regions. As a whole, the energy fluxes, as well as the average electron energy, began to grow during the substorm growth phase and reached the maximum level at the end of the substorm expansive phase. The most essential increase in energy flux (more than ten times) this time was observed in the most equatorward precipitation region $(b 1 e-b 2 i)$, corresponding to the region of diffuse auroral luminosity. Just before the beginning of the substorm expansive phase, the energy flux in all regions remained about constant with the exception of the $b 4 s-b 5$ region, where the flux increase of about 1.5 times was registered. 

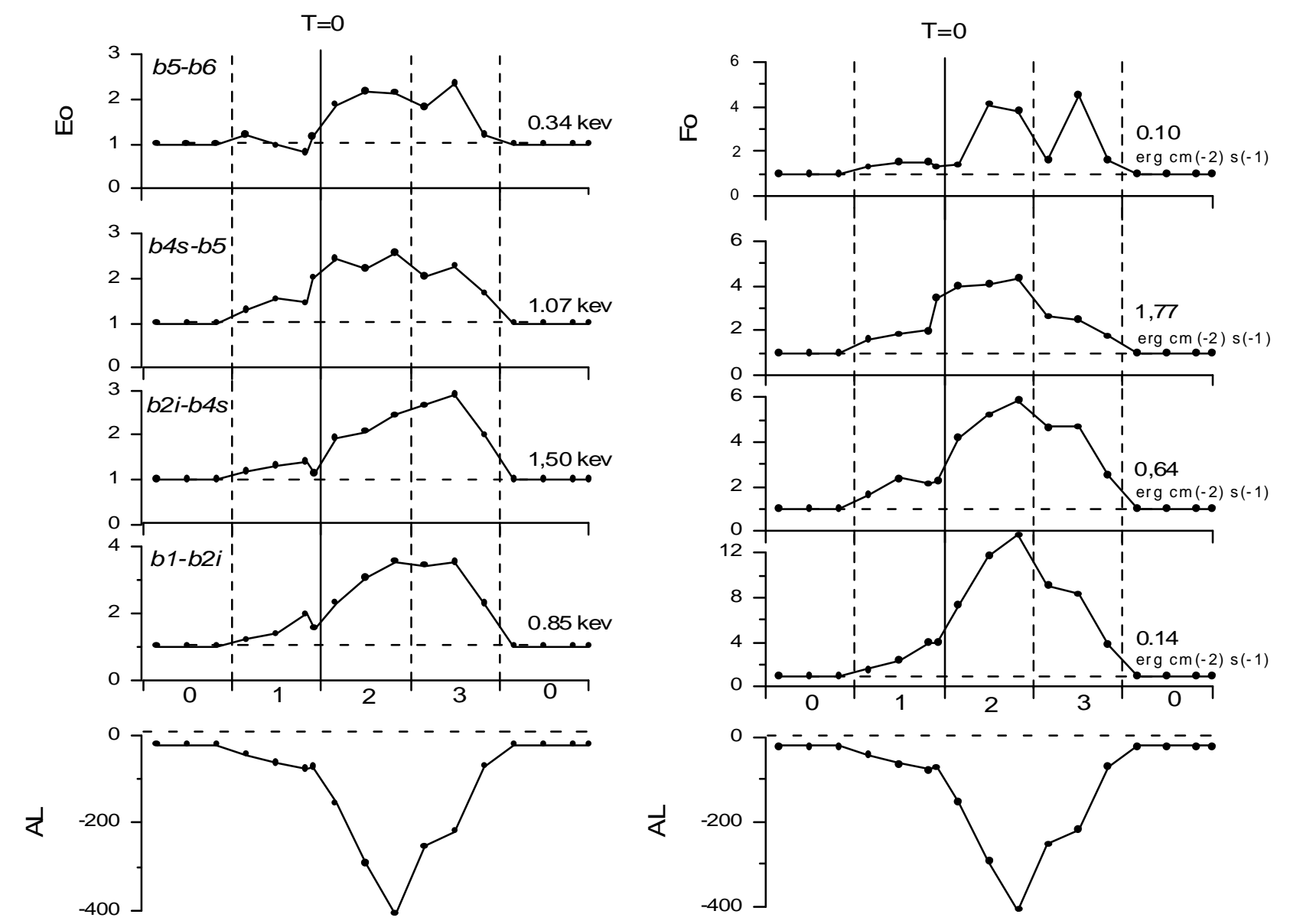

Fig. 3. The average electron precipitation energy (a) and energy flux (b) in various precipitation regions during the substorm. They are standardized to those during magnetic quietness, which absolute levels are shown in the right part of figures (a) and (b). The label of the regions is marked in the left part of figure (a). The bottom panel demonstrates the average $A L$ index variation.

\section{Discussion}

The analysis of interplanetary medium conditions during substorms is extremely important for finding out whether the magnetospheric substorm is a consequence of internal magnetospheric instabilities or whether it is triggered by sharp changes in the interplanetary parameters (Lyons, 1996 and references here). The general external sources of the substorm triggering can be a sharp decrease or a northern turn of the southward IMF $B_{z}$ component (Caan et al., 1978; Dmitrieva and Sergeev, 1983), impulsive changes in the solar wind dynamic pressure (Kokubun et al., 1977; Sauvaud, 1998) and sharp variations in the IMF $B_{y}$ component (Dmitrieva and Sergeev, 1983; Troshichev et al., 1986). So, Lyons (1996) considered that the substorm expansive phase is caused generally by external sources. On the other hand, from statistical data Maltsev (1998) showed that the substorm onset was neither associated with sharp $\left|B_{z}\right|$ changes, nor with solar wind dynamic pressure variations, that, in the author's opinion, testifies to the spontaneous nature of substorms.

Our statistical results show that although, on average, dur- ing the substorms, the solar wind dynamic pressure was a little bit higher than during quietness periods, at the time of the substorm onset any essential or sharp changes in the dynamic pressure were not observed. The substorm expansive phase began generally during the southward IMF orientation and, on average, the substorm onset was not associated with a decrease or a northern turn of the southward interplanetary $B_{z}$. In this point our conclusions coincide with the results obtained by Maltsev (1998). The IMF $B_{y}$ component behavior is of interest. As one can see from Fig. 1, in the winter season substorms were observed predominantly during the positive $B_{y}$ orientation and in summer when the $B_{y}$ was negative. A similar result had been earlier obtained by Vorobjev and Zverev (1982). It is important to note here that, according to our data, the most essential changes in the interplanetary $B_{y}$ orientation were registered after the substorm onset. It is well known (Sibeck et al., 1989; Fairfield et al., 1987; Vorobjev et al., 1999) that a change in the IMF $B_{y}$ orientation results in a change in the geometry foreshock upstream from the Earth's bowshock, that in turn, can be accompanied by sharp changes in the dynamic pressure in the sunlit magnetopause. Moreover, Vorobjev et al. $(2001,2002)$ showed that 


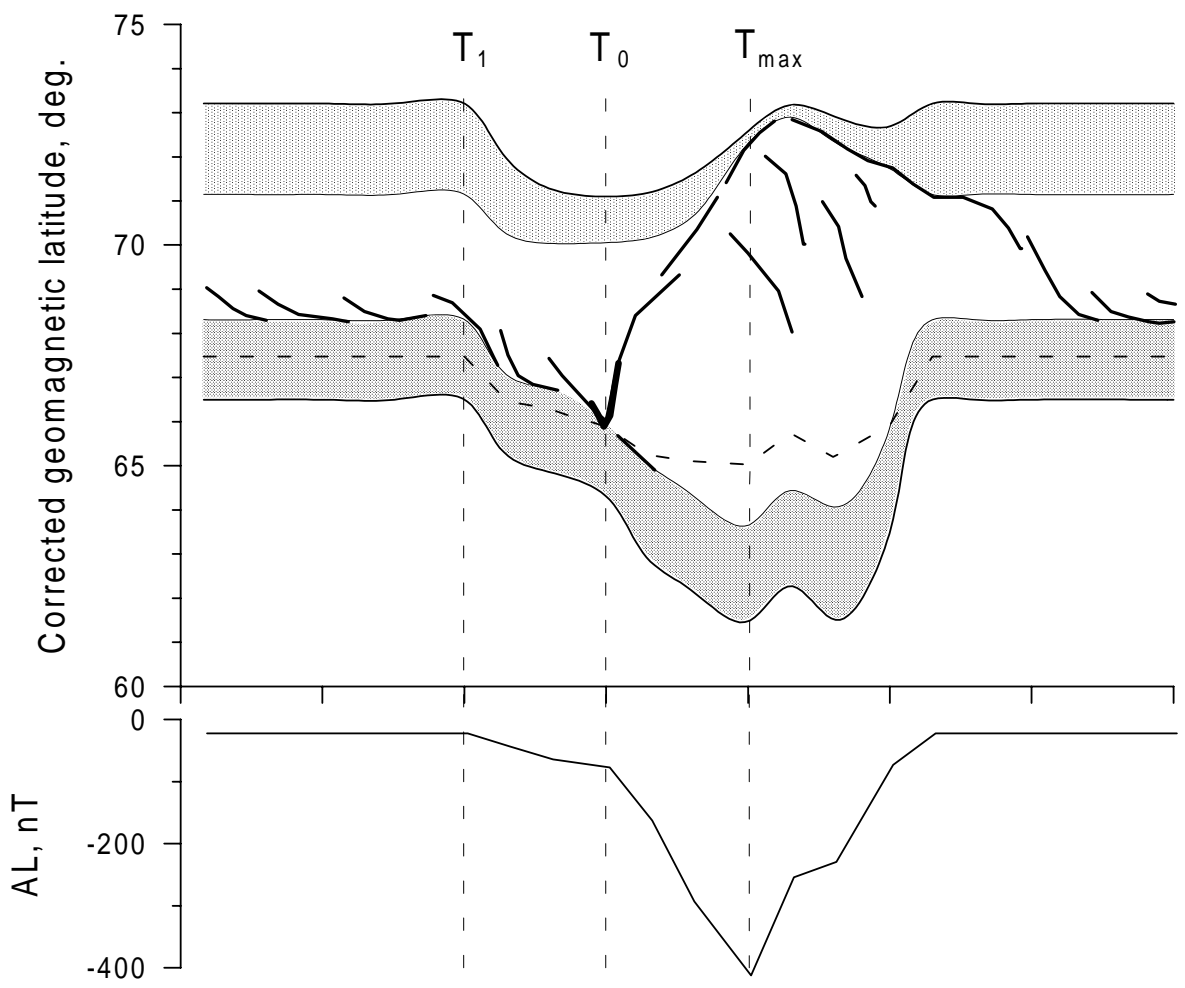

Fig. 4. The summarized scheme of the substorm development in the auroral precipitation and discrete aurorae. Dark areas are regions of diffuse precipitation border of the "auroral oval precipitation" region. The ion isotropy boundary is shown by the dashed line and dynamics of discrete aurorae by segments of heavy lines.

the $B_{y}$ polarity during a substorm development essentially affects both the midnight latitudinal position of the poleward electron precipitation boundaries ( $b 5$ and $b 6)$ and the maximum latitude of the auroral bulge. In this way, sharp changes in the $B_{y}$ orientation in an ecliptic plane can be one of the important factors that influence the development of magnetospheric disturbances.

The width of the auroral precipitation in the midnight sector (Fig. 2) during the period of magnetic quietness was about $6.5^{\circ}$ of latitude and increased up to $11^{\circ}$, to the maximum of the substorm expansive phase of about $\sim 400 \mathrm{nT}$ in intensity. This expansion occurred generally due to the displacement of the equatorward precipitation boundary $(b 1 e)$ to lower latitudes. In contrast with this, the width of the auroral oval during quiet periods is only $\sim 2-3^{\circ}$ of the latitude (Starkov and Feldstein, 1967). It is well known (Akasofu, 1964) that a substorm usually begins by a sudden growth of intensification of the auroral arc at the equatorward border of the auroral oval and is accompanied by the subsequent fast poleward displacement of bright auroral forms. Samson et al. (1992) showed that the auroral intensification at the substorm onset had occurred in the region of intensive proton penetration. Since the $b 2 i$ boundary was determined as the latitude where the energy flux of ions had the maximum, the auroral breakup most likely occurs near the region of the $b 2 i$ and $b 2 e$ boundaries' crossing. The width of an auroral arc is a few $\mathrm{km}$ at most, while the width of the auroral precipitation re- gion to the pole from the $b 2 i$ and $b 2 e$ crossing is about $5^{\circ}$ of latitude. So, one can suggest that the auroral break-up occurs deeply in the magnetosphere in the transition region where the magnetic field lines change from dipole-like to extended ones in the tail. This result coincides with the conclusions in the paper of Samson et al. (1992), where it is shown by scanning photometer data that the initial auroral intensification occurs at field lines that are about $5^{\circ}$ of latitude equatorward of the polar cap boundary and that cross the equatorial plane of the magnetosphere at 6-10 $R_{E}$.

In the paper by Starkov et al. (2002) a new notation for different precipitating regions observed with the DMSP satellite was suggested. It was carried out on the basis of a statistical comparison of the auroral precipitation structure with the boundaries of the auroral oval and diffuse auroral luminosity, and allows one to describe uniformly the auroral phenomena, both in the day and night sectors. The main regions of precipitating particles in this notation are: "diffuse auroral zone" (DAZ) - the precipitation between the $b 1 e-b 2 e$ boundaries; "auroral oval precipitation" (AOP) - between the $b 2 e-b 5$ boundaries and "soft diffuse precipitation" (SDP) - between the $b 5$ and $b 6$. The summarized scheme of the substorm development in the auroral precipitations and discrete aurorae is shown in Fig. 4 using this notation. Corrected geomagnetic latitudes are marked along the vertical axis. The regions of diffuse precipitations (dark areas) border on the equatorward and poleward edges of the "auroral oval 
precipitation" region. The ion isotropy boundary is shown by the dashed line, and dynamics of discrete aurorae by segments of heavy lines. To construct the auroral forms' dynamics, the results published by Akasofu (1964), Starkov and Feldstein (1971), Snyder and Akasofu (1971), Craven and Frank (1990), Samson et al. (1992) and others are used. The beginning time of the substorm growth phase, the substorm onset, and the maximum in the substorm development are marked by symbols $T_{1}, T_{0}$ and $T_{\max }$, respectively. In this scheme the main development of aurorae occurs in the "auroral oval precipitation" region and thus, the location of the poleward boundary of discrete aurorae does not determine the polar cap boundary. Interesting is the decrease in the average electron precipitation energy in the DAZ and in the equatorward part of the AOP region, about 5 min before $T=0$. This energy decrease can correspond to the fading of discrete aurorae and diffuse luminosity just before the beginning of the substorm (Zaitseva et al., 1976; Pellinen and Heikkila, 1978; Kornilova et al., 1989). Simultaneously with this energy decreasing in the equatorial part of auroral zone, the enhancement in both the average electron precipitation energy and energy flux was observed in the poleward part of the AOP region. The energy flux increase was about 1.5 times compared to their average level at the final stage of the substorm growth phase. The increase in both the average auroral electron energy and energy flux in this region testifies to the fact that, about $5 \mathrm{~min}$ before the substorm onset time, it is possible to expect an increase in auroral luminosity or occurrence of visual aurorae at CGLs of about $67^{\circ}-71^{\circ}$, i.e. a few degrees poleward of the bright discrete aurorae of the auroral oval (substorm precursor).

This result can be indirect evidence of the substorm mechanism suggested by Shiokava et al. (1997) and Reeves (1998). In their "braking model" the formation of a neutral line in the magnetospheric tail precedes the beginning of the substorm expansive phase determined by auroral break-ups, Pi2 bursts, the sharp beginning of magnetic bays, the appearance of particle injections at geosynchronous distances, etc. The beginning of the reconnection produces fast convective plasma flows that are directed earthward and tailward from the reconnection region. When the strong flows approach the dipole-like inner magnetosphere, they are forced to slow down and divert around the Earth.

The braking process of the flow could cause an azimuth pressure gradient, a strong inductive electric field, and vortical flows, that, in turn, produce the field-aligned currents of the substorm current wedge. The compression pulses and fluctuations of the field-aligned currents generated in the braking point can be an initial cause of the Pi2 magnetic pulsations, the particle injections into the inner magnetosphere and the appearance of other ground features of a substorm onset. Actually, Nagai et al. (1998) showed that magnetic reconnection most likely takes place in the limited area of the near-Earth plasma sheet at the distances of 20$30 R_{E}$, downtail or closer to the Earth (Mukai et al., 1998; Machida et al., 1998), a few minutes prior to the substorm onsets which were identified by the Pi2 appearance. It suggests that at least in some particular cases the reconnection triggers the substorm. In the papers by Fairfield et al. $(1998,1999)$ there were examples of high speed flows observed with the Geotail satellite that were clearly associated with substorms and, in fact, preceded the auroral break-ups and substorm injections. Thus, the possibility cannot be excluded that some substorms can be initiated by the magnetic reconnection in the tail and the high-speed flow occurrence. However, we shall note that the analysis of optical data presented by Yahnin et al. (2000) did not discover any auroral precursor for several selected substorms.

\section{Conclusions}

DMSP F7 satellite observations for the whole of 1986 in the magnetic local time interval from 21:00 to 24:00 MLT were used to examine the different auroral precipitation boundary dynamics and precipitating electron features during all substorm phases. The general results can be summarized as follows:

1. The empirical model of the substorm in the midnight auroral precipitation is suggested. The model includes dynamics of different electron precipitation boundaries during all substorm phases together, with simultaneous changes in average electron precipitation energy and energy flux in different precipitation zones, as well as the IMF and solar wind plasma behavior during the substorm.

2. The analysis of observations showed that during magnetic quietness and just before the beginning of the substorm expansive phase, the latitudinal width of the auroral precipitation in the nightside sector is about $6-7^{\circ} \mathrm{CGL}$.

3. For about $5 \mathrm{~min}$ prior to the substorm onset time a decrease in the average precipitating electron energy in the equatorward part of auroral zone was observed simultaneously with an increase in both the average precipitating electron energy and energy flux in the poleward part of the auroral precipitation zone.

4. The isotropy boundary position in the beginning of the substorm expansive phase $(T=0)$ coincides with the inner edge of the central plasma sheet. During magnetic quietness and the substorm growth phase, the isotropy boundary is placed closer to the Earth than the central plasma sheet, but, in contrast, it is inside the main plasma sheet during the period of the substorm expansive phase and magnetic disturbances.

5. On average, during the substorm, the solar wind dynamic pressure was about 1.5 times that of the magnetic quietness period. Substorms occurred predominantly during the southward IMF orientation, and the substorm onset is not often associated with the northern 
turn or with the decrease in the southward interplanetary $B_{z}$. In winter substorms occurred generally during the positive interplanetary $B_{y}$ and in summer they were observed when the interplanetary $B_{y}$ was negative.

Acknowledgements. The DMSP particle detectors were designed by Dave Hardy of AFRL, and data obtained from JHU/APL. We thank Dave Hardy, Fred Rich, and Patrick Newell for its use. We thank Drs. J. H. King and N. Papitashvili for providing IMF and solar wind plasma observations in the NSSDC OMNIWeb database. The WDC for geomagnetism in Kioto provided magnetic activity indexes. The paper was supported by the RFBR grants 02-05-64807 and 99-05-65611, INTAS-2000-752 and ISSI, Bern.

Topical Editor G. Chanteur thanks two referees for their help in evaluating this paper.

\section{References}

Akasofu, S.-I.: The development of the auroral substorm, Planet. Space Sci., 12, 273, 1964.

Akasofu, S.-I., Meng, C.-I., and Kimball, D. S.: Dynamics of aurora. 4. Polar magnetic substorm and westward traveling surge, J. Atmos. Terr. Phys., 28, 489, 1966.

Caan, M. N., McPherron, R. L., and Russell, C. N.: The statistical magnetic signature of magnetospheric substorm, Planet. Space Sci., 26, 269, 1978.

Craven, J. D. and Frank, L. A.: Latitudinal motion of the aurora during substorm, J. Geophys. Res., 95, 3773, 1990.

Dmitrieva, N. P. and Sergeev, V. A.: Spontaneous and compelled beginning of a burst phase of the magnetospheric substorm and its preliminary phase duration, Geomagn. Aeron. (in Russian), 23, 470, 1983.

Fairfield, D. H., Baumjohann, W., Paschmann, G., Luhr, H., and Sibeck, D. G.: Upstream pressure variations associated with the bow shock and their effects on the magnetosphere, J. Geophys. Res., 92, 4565, 1987.

Fairfield, D. H., Mukai, T., Lui, A. T. Y., et al.: Geotail observation of substorm onset in the inner magnetotail, J. Geophys. Res., 103, 103, 1998.

Fairfield, D. H., Mukai, T., Yamomoto, T., et al.: Earthward flow bursts in the inner magnetotail and their relation to auroral brightenings, AKR intensifications, geosynchronous particle injections and magnetic activity, J. Geophys. Res., 104, 355, 1999.

Feldstein, Ya. I. and Galperin, Y. I.: Structure of the auroral precipitations in the nightside sector of the magnetosphere, Cosmic Research, (in Russian), 34, 227, 1996.

Hardy, D. M., Gussenhoven, M. S., and Holeman, E. A.: Statistical model of auroral electron precipitation, J. Geophys. Res., 90, 4229, 1985.

Iijima, T. and Nagata, T.: Signatures for the substorm development of the growth phase and expansion phase, Planet. Space Sci., 20, 1095, 1972.

Kokubun, S.: Polar substorm and interplanetary magnetic field, Planet. Space Sci., 18, 697, 1970.

Kokubun, S., McPherron, R. L., and Russell, C. T.: Triggering of substorms by solar wind discontinuities, J. Geophys. Res., 82, 74, 1977.

Kornilova, T. A., Pudovkin, M. I., and Starkov, G. V.: Aurora damping before a breakup, Geomagn. Aeron., (in Russian), 29, 503, 1989.
Lyons, L. R.: Fundamental observational features, distinction from other disturbances, and external triggering, J. Geophys. Res., 101, 13 011, 1996.

McPerron, R. L.: Growth phase of magnetospheric substorm, J. Geophys. Res., 75, 5592, 1970.

Machida, S., Miyashita, Y., Ieda, A., et al.: Time evolution of the Earth magnetotail associated with substorm onset: Geotail observations, in: Substorm-4, edited by Kokubun, S. and Kamide, Y., Astrophysics and Space Science Library, Japan, 238, 149, 1998.

Maltsev, Yu. P.: Search of relation between the substorm onset and the solar wind parameters, in: Substorm-4, edited by Kokubun, S. and Kamide, Y., Astrophysics and Space Science Library, Japan, 238, 291, 1998.

Mukai, T., Hoshino, M., Saito, Y., et al.: Pre-onset and onset signatures for substorms in the near-tail plasma sheet: Geotail observations, in: Substorm-4, edited by Kokubun, S. and Kamide, Y., Astrophysics and Space Science Library, Japan, 238, 131, 1998.

Nagai, T., Fujimoto, M., Saito, Y., et al.: Structure and dynamics of magnetic reconnection for substorm onset with Geotail observations, J. Geophys. Res., 103, 4419, 1998.

Newell, P. T., Feldstein, Ya. I., Galperin., Yu. I., and Meng, C.-I.: Morphology of nightside precipitation, J. Geophys. Res., 101, $10737,1996$.

Newell, P. T., Sergeev, V. A., Bikkuzina, G. R., and Wing, S.: Characterizing the state of the magnetosphere: testing the ion precipitation maxima latitude (b2i) and the ion isotropy boundary, J. Geophys. Res., 103, 4739, 1998.

Pellinen, R. J. and Heikkila, W. J.: Observation of auroral fading before breakup, J. Geophys. Res., 83, 4207, 1978.

Reeves, G. D.: New perspectives on substorm injections, in: Substorm-4, edited by Kokubun, S. and Kamide, Y., Astrophysics and Space Science Library, 238, Japan, 785, 1998.

Samson, J. C., Lyons, L. R., Xu, B., Creutzberg, F., and Newell, P.: Proton aurora and substorm intensifications, Geophys. Res. Lett., 19, 2167, 1992.

Sauvaud, J. A.: An attempt to characterize substorm drivers in the interplanetary medium, in: Substorm-4, edited by Kokubun, S. and Kamide, Y., Astrophysics and Space Science Library, Japan, 238, 131, 1998.

Shiokava, K., Baumjohann, W., and Haerendel, G.: Braking of the high-speed flows in the near-Earth tail, Geophys. Res. Lett., 24, 1179, 1997.

Sibeck, D. G., Baumjohann, W., Elphic, R. C., et al.: The magnetospheric response to 8-minute period strong-amplitude upstream pressure variations, J. Geophys. Res., 94, 2505, 1989.

Snyder, A. L. and Akasofu, S.-I.: Observation of auroral oval by the Alaskan meridian chain of stations, J. Geophys. Res., 77, 3419, 1972.

Sotirelis, T. and Newell, P. T.: Boundary-oriented electron precipitation model, J. Geophys. Res., 105, 18 655, 2000.

Spiro, R. W., Reiff, Jr., P. H., and Maher, L. J.: Precipitating electron energy flux and auroral zone conductances - an empirical model, J. Geophys. Res., 87, 8215, 1982.

Starkov, G. V. and Feldstein, Ya. I.: Variation of auroral oval zone boundaries, Geomagn. Aeron., (in Russian), 7, 62, 1967.

Starkov, G. V. and Feldstein, Ya. I.: Substorm in aurorae, Geomagn. Aeron., (in Russian), 11, 560, 1971.

Starkov, G. V., Rezhenov, B. V., Vorobjev, V. G., Feldstein, Ya. I., and Gromova, L. I.: Structure of the auroral precipitations in the dayside sector, Geomagn. Aeron. (in Russian), 42, 186, 2002.

Troshichev, O. A., Kotikov, A. L., Bolotinskaya, B. D., and Andrezen, V. G.: Influence of the IMF azimuthal component on the 
magnetospheric substorm dynamics, J. Geomag. Geoelectr., 38, 1075, 1986.

Vorobjev, V. G. and Zverev, V. L.: Influence of solar wind parameters on geomagnetic activity, Geomagn. Aeron., (in Russian), 22, 257, 1982.

Vorobjev, V. G., Yagodkina, O. I., and Zverev, V. L.: Morphological features of bipolar magnetic impulsive events and associated interplanetary medium signatures, J. Geophys. Res., 104, 4595, 1999.

Vorobjev, V. G., Gromova, L. I., Rezhenov, B. V., Starkov, G. V., and Feldstein, Ya. I.: Variations of the boundaries of plasma precipitation and auroral luminosity in the night-time sector, Geomagn. Aeron., (in Russian), 40, 79, 2000.

Vorobjev, V. G., Yagodkina, O. I., Sibeck, D., Liou, K., and Meng,
C.-I.: Auroral conjugacy during substorm: Coordinated Antarctic ground and Polar Ultraviolet observations, J. Geophys. Res., 106, 24 579, 2001.

Vorobjev, V. G., Yagodkina, O. I., Starkov, G. V., and Feldstein, Ya. I.: Influence of the IMF sector structure on the locations of auroral precipitation boundaries in the night-time sector, Geomagn. Aeron., (in Russian), 42, 477, 2002.

Yahnin, A. G., Kornilov, I. A., Kornilova, T. A., et al.: Do the observations confirm the high-speed flow braking model for substorms? Fifth international conference on substorm, edited by Wilson, A., European Space Agency, SP-443, 345, 2000.

Zaitseva, S. A., Kuznetsov, B. M., and Pudovkin, M. I.: Auroral and current dynamics in the course of substorm progress, Geomagn. Res., (in Russian), 18, 73, 1976. 